\title{
Efecto de diferentes niveles dietéticos de harina de pescado sobre la producción y calidad de huevos de codornices
}

\section{Effect of different dietary levels of fish flour on the production and quality of eggs quails}

José Gregorio Pino Universidad Politécnica Territorial del Oeste de Sucre "Clodosbaldo Russian" Cariaco (Venezuela)

Enrique Pino Hernández Centro de Engenharia Biológica, Universidade do Minho, Campus de Gualtar, Braga, Portugal eph@ceb.uminho.pt

Pedro Manuel Villa Fundación para la Conservación de la Biodiversidad, Puerto Ayacucho

(Venezuela)

Jesús Ruiz-González Departamento de Engenharia de Alimentos, Universidade Federal de Viçosa, Minas Gerais

Revista Cumbres Vol.4 №2

Versión impresa ISSN 1390-9541

Versión electrónica ISSN 1390-3365

http://investigacion.utmachala.edu.ec/revistas/index.php/Cumbres 


\section{RESUMEN}

El uso de la harina de pescado como suplemento nutricional en las dietas suministradas a codornices fue estudiado para identificar el efecto sobre la producción y calidad del huevo. Se analizaron 180 codornices bajo un diseño experimental completamente al azar, con seis tratamientos, tres repeticiones y 10 codornices por unidad experimental. Se utilizaron dietas experimentales considerando diferentes niveles de adición de harina de pescado $(0 \%, 6 \%$, $7 \%, 8 \%, 9 \%$ y $10 \%$ ) a un alimento comercial. Fueron analizadas diferentes variables de producción y calidad del huevo de codorniz: número de huevos, peso, volumen, manchado, brillantez y huevos rotos. Así como, la composición proximal y la calidad sensorial. Se utilizó ANOVA y test de Tukey al 5\% para el análisis estadístico de los datos. Hubo diferencia significativa entre tratamientos con la variable número de huevos. Las variables peso y volumen no mostraron diferencias significativas. Sin embargo, el manchado y brillantez mostraron diferencias entre los tratamientos, cuyo valor óptimo fue alcanzado por el tratamiento con 9\% de harina de pescado. Estos resultados evidencian que existe una influencia positiva de la adición de 9\% de harina de pescado sobre la calidad del manchado, brillantez del huevo de codorniz y sobre la composición nutricional. Del mismo modo, los atributos sensoriales (olor y sabor) de los huevos fueron aceptables. Los resultados indican que los huevos de codorniz obtenidos del T9 son adecuados para la reproducción artificial.

Palabras clave: proteína animal; reproducción artificial; producción de huevos; calidad de los huevos.

\section{ABSTRACT}

The use of fish flour as a nutritional supplement in diets provided to quail was studied to identify the effect on egg production and its quality. For this study, 180 quail were analyzed under a completely randomized experimental design, with six treatments, three repetitions and 10 quails per experimental unit. Experimental diets were used considering different levels of fish flour addition $(0 \%, 6 \%, 7 \%, 8 \%, 9 \%$ and $10 \%)$ to commercial feed. ANOVA and Tukey test at $5 \%$ were used to made a statistical analysis of the data. Different variables of egg production and its quality were analyzed: number of eggs, weight, volume, spotting, brilliance and broken eggs, as well as, the proximal composition and sensory quality. Significant differences were identified between treatments when the variable number of eggs was considered. The variables weight and volume did not show significant differences. However, spotting and brilliance showed differences between treatments, where the optimal value was reached by treatment with $9 \%$ of fish flour. This result shows that there is a positive influence of the addition of $9 \%$ of fishmeal on the quality of spotting and brilliance of the quail egg, and on the nutritional 
composition. Similarly, the sensory attributes (odor and flavor) of the eggs were acceptable. The results indicate that quail eggs obtained from T9 are suitable for artificial reproduction.

Keywords: animal protein; artificial reproduction; eggs production; egg quality.

\section{INTRODUCCIÓN}

La codorniz (Coturnix coturnix) es un ave originaria de Europa, Norte de África y Asia, y ha sido identificado como un animal rustico y resistente a enfermedades. Su carne tiene poco contenido de grasa, y principalmente sus huevos son muy apreciados por su valor nutricional (Lucotte, 1985). Esta ave se considera uno de los productos avícolas con mayor oferta y demanda en el mercado nacional e internacional, que a su vez es recomendado por pediatras y geriatras, para incluirla en la dieta de niños y ancianos (Pérez y Pérez, 1974). Por otra parte, estas aves son de crecimiento rápido, alta precocidad en la producción y madurez sexual (35 a 42 días), una alta productividad de huevos (media de 300 huevos/año) en pequeños espacios, alta longevidad con un alto rendimiento (14 a 18 meses), además de la baja inversión en los sistemas de producción (Pinto et al., 2002).

La alimentación de las codornices con fines productivos depende del alimento concentrado que, generalmente no es de fácil obtención por la limitación de materia prima para las industrias. Por lo que muchas veces es reemplazado por alimento de gallinas ponedoras. En este sentido, codornices con dietas que no cumplen con las propiedades nutricionales que este tipo de aves demanda, han mostrado serios trastornos digestivos y reproductivos, que retardan el desarrollo, disminuyen la postura e incluso ocasionan susceptibilidad a enfermedades y hasta la muerte de las mismas. Así mismo, se ha reportado que un contenido de proteína y desbalances en los aminoácidos esenciales en dietas para codornices trae como consecuencias una disminución y hasta la supresión de la postura (Hurtado-Nery et al., 2010; Lima et al., 2014). Además de la demanda de proteína en la alimentación de la codorniz, también son indispensables otros compuestos nutritivos balanceados como hidratos de carbono, grasas, vitaminas, minerales y aminoácidos esenciales, según sus etapas fisiológicas (Ribeiro et al., 2015).

Frente a la necesidad de desarrollar alternativas sustentables que permitan complementar el componente proteico requerido para la dieta de las codornices, se ha considerado el uso de recursos locales de origen animal y vegetal (Oliveira et al., 2015; Pereira et al., 2016; Rosario y Nieves, 2015). En este sentido, con el suministro equilibrado entre el alimento concentrado comercial y el porcentaje correcto de harina de pescado, se espera disminuir las pérdidas por la mala calidad de la cáscara del huevo y alcanzar el mejoramiento en la calidad del huevo, que puede resultar en un progreso significativo para la industria en un mercado cada vez más expansivo y al mismo tiempo, competitivo. Según Hurtado-Heny et al. (2010) la demanda de energía requerida para mantener el alto régimen de postura y el elevado 
peso de los huevos, obliga a que la dieta de las codornices tenga un alto valor proteico. Por esta razón, la harina de pescado, se presenta como una materia prima alternativa que puede registrar una fuente de energía concentrada entre 70 y 80 \% en forma de proteína, conteniendo además minerales y vitaminas en cantidades importantes (Oliveira et al., 2015).

Según Quitral et al. (2009) posiblemente el efecto negativo encontrado sensorialmente en huevos de aves alimentadas con dietas que contienen harina de pescado se debe a la presencia de trimetilamina, la que se forma por degradación microbiana y por alteración de nitrógeno básico volátil total (N-BVT). Por otra parte, las dietas con presencia de aceite de pescado y donde las muestras de huevo registren sabores no característicos al producto, probablemente se deba a la peroxidación lipídica, es decir, alteración en especies reactivas del oxígeno (Thiobarbituric acid reactive substances - TBARS). Pino Hernández et al. (2017) hace referencia de N-BVT y TBARS de 2,00 \pm 0,03 (mg/100 g) y 0,40 \pm 0,04 (mgMDA/kg) respectivamente, en musculo de pescado fresco, teniendo esos valores como referencia aceptable.

El objetivo de este trabajo fue evaluar el efecto de la harina de pescado como complemento nutricional en las dietas suministradas a codornices. Cabe destacar que, la harina de pescado es un subproducto de la industria pesquera y con su utilización se contribuye a la reducción de residuos y a la de los costos de producción; además, se incrementa la disponibilidad de huevos y carne de codorniz para las poblaciones locales.

\section{MATERIALES Y MÉTODOS}

El estudio se realizó en la Universidad Politécnica Territorial del Oeste de Sucre "Clodosbaldo Russián”, de Cariaco estado Sucre, Venezuela (10 30' $00^{\prime \prime} \mathrm{N}$ y 68 $38^{\prime} 12^{\prime \prime} \mathrm{O}$ ) a una altitud de $8 \mathrm{msnm}$. El área de trabajo pertenece a la zona de la fosa tectónica Cariaco-Casanay el clima es cálido y subhúmedo, presentando una temperatura promedio anual de $27^{\circ} \mathrm{C}$, precipitación mensual promedio de $900 \mathrm{~mm}$ y una humedad relativa que fluctúa alrededor de $86 \%$ (INE, 2011).

El diseño experimental para este estudio comprendió la selección de 180 codornices en postura de 22 semanas de edad, con un peso promedio de 120 $\pm 3,8 \mathrm{~g}$, las cuales fueron distribuidas en jaulas (previamente desinfectadas e identificadas) considerando un diseño experimental completamente al azar, con seis tratamientos, tres repeticiones y 10 codornices por unidad experimental. Las jaulas consistieron de alambre galvanizado con dimensiones de 0,90 x 0,80 x 0,25 m y una inclinación entre el 10 y 15\%, para lograr que los huevos se deslicen hasta la parte exterior de la jaula. Las jaulas estaban provistas de comedores lineales de lámina galvanizada y bebederos automáticos tipo sifón. Se establecieron bloques conformados por tres jaulas, una encima de la otra.

Las dietas experimentales fueron formuladas para obtener raciones balanceadas atendiendo los requerimientos alimenticios y nutricionales de codornices en postura. En este sentido, fue mezclado manualmente alimento comercial con harina de pescado en la siguiente composición de proteínas

\section{0}


(75,00 \%), lípidos (6,05\%), humedad (13,08 \%) y ceniza (4,07 \%). Esta fuente de origen animal, fue obtenida de residuos de la industria pesqueras, los cuales fueron sometidos a un proceso de secado a $60{ }^{\circ} \mathrm{C}$ en estufa de aire forzado (DeLeo, model Q 314 M122) y posteriormente a una molienda. Las características finales, fue un producto rico en proteínas y con parámetros de calidad de N-BVT y TBARS de 1,95 \pm 0,13 (mg/100 g) y 0,31 \pm 0,09 (mgMDA/kg), respectivamente. Las concentraciones de harina de pescado utilizadas fueron 0\%, 6\%, 7\%, 8\%, 9\% y $10 \%$ por cada $100 \mathrm{Kg}$. Las dietas se mezclaron manualmente y fueron suministradas en forma de harina (tabla 1), estas registraron valores de 0,55 - 1,10 y 0,95 - 3,33 \% de fosforo y calcio, respectivamente.

\begin{tabular}{|lll|l|l|l|l|l|}
\hline \multirow{2}{*}{ INGREDIENTES (\%) } & \multicolumn{1}{l}{ TRATAMIENTOS } \\
\cline { 2 - 8 } & T1 & T6 & T7 & T8 & T9 & T10 \\
\hline Maíz molido & 50,0 & 47,0 & 46,5 & 46,0 & 45,5 & 45,0 \\
\hline Torta de soja & 30,0 & 28,2 & 27,9 & 27,6 & 27,3 & 27,0 \\
\hline Harina de sorgo & 13,0 & 12,2 & 12,1 & 12,0 & 11,8 & 11,7 \\
\hline Carbonato de calcio & 0,50 & 0,50 & 0,50 & 0,50 & 0,50 & 0,50 \\
\hline Cloruro de sodio & 0,50 & 0,50 & 0,50 & 0,50 & 0,50 & 0,50 \\
\hline Harina de carne & 2,00 & 1,90 & 1,90 & 1,80 & 1,80 & 1,80 \\
\hline Aceite vegetal & 4,00 & 3,80 & 3,70 & 3,70 & 3,60 & 3,60 \\
\hline Harina de pescado & 0,00 & 6,00 & 7,00 & 8,00 & 9,00 & 10,0 \\
\hline Total & 100 & 100 & 100 & 100 & 100 & 100 \\
\hline
\end{tabular}

Tabla 1. Formulaciones de los tratamientos establecidos con diferentes dosis de harina de pescado.

El experimento se realizó durante dos meses, incluida una semana de adaptación a las dietas experimentales y siete semanas de evaluación de las variables asociadas al comportamiento productivo, calidad y composición del huevo. La ración ofrecida fue aproximadamente 29 g/ave/día en dos suministros; 09:00 h y 17:30 h, con el propósito de garantizar un consumo ad libitum por parte de las aves y el suministro de agua a lo largo de todo el experimento. La limpieza de los depósitos de agua se realizó diariamente, mientras que, las bandejas de colectas de heces se limpiaron cada tres días. Fueron analizadas diferentes variables de producción y calidad del huevo de codorniz: número de huevos, peso, volumen, manchado y brillantez.

El número de huevos producidos fue registrado diariamente y recolectados para sus respectivos análisis. Seguidamente los huevos se clasificaron entre rotos o con defectos en la cascara, y comercializables.

La estimación proporcional de los huevos producidos por tratamiento se efectuó empleando la ecuación 1, la cual permite determinar el porcentaje de postura. Así mismo, se determinó el porcentaje de huevos comercializables.

$$
\% \text { Postura }=\left(\frac{\mathrm{N}^{\circ} \text { de huevos producidos }}{\mathrm{N}^{\circ} \text { de aves en jaula }}\right) \times 100
$$


La cantidad de alimentos, medido en gramos, fue obtenido a través de la diferencia, peso de inicio (ración) y peso final (sobra).

El peso de los huevos fue determinado usando una balanza digital (77014, KERN, Alemania) y la relación presentada en la ecuación 2 :

$$
\mathrm{Ph}=\left(\frac{\text { Kg de huevos }}{\mathrm{N}^{\circ} \text { de huevos }}\right)
$$

(Ecuación. 2)

Donde, $\mathrm{Ph}=$ Peso de los huevos; Kg. de huevos $=\Sigma$ de todos los pesos de los huevos muestreados/semana; № Huevos = número de huevos muestreados.

El volumen de los huevos fue medido utilizando un vernier mecánico con una precisión de 0,1 $\mathrm{mm}$, midiéndose semanalmente tanto la altura como el diámetro de una muestra de 5 huevos por repetición, resultando un total de 15 huevos por tratamiento.

La caracterización cualitativa se realizó para el 5\% de la muestra de huevos comercializables por cada tratamiento, a través de observaciones de parámetros color y brillo. Para el color de la cáscara se consideró presencia de manchas o pigmentación (poco manchados, medianamente manchados y muy machados) y para el brillo de la cáscara se consideró dos cualidades, opacas y brillantes.

El análisis bromatológico se realizó para determinar el contenido porcentual de proteína, lípidos, fibra y cenizas en los diferentes tratamientos y en los huevos (tratamiento $100 \%$ alimento comercial y mezcla de los tratamientos con harina de pescado) de acuerdo a la metodología descrita en la AOAC (2000).

El análisis sensorial se realizó para evaluar el efecto de la inclusión de la harina de pescado en la dieta de las codornices. El tiempo de cocción de las muestras fue establecido por ensayos preliminares, estableciendo así, 10 minutos contados a partir de la ebullición del agua. Inmediatamente después de la cocción, cada panelista recibió un huevo de cada tratamiento. A los evaluadores se les pidió usar galletas con poca sal y agua para limpiar su paladar entre las muestras evaluadas. La evaluación se realizó en cabinas individuales bajo condiciones controladas de luz, temperatura y humedad. La prueba de aceptación se llevó a cabo con 100 consumidores (de 20 a 50 años), preseleccionados de acuerdo a los intereses y hábitos de consumo de huevo de codorniz. Se utilizó escala hedónica que variaba de 1 (me disgusta mucho) a 9 (me gusta extremadamente), evaluando los siguientes atributos color, olor, textura, sabor y apreciación general (Dutcosky, 2013).

Los resultados de los parámetros evaluados fueron analizados estadísticamente a través de un análisis de varianza (ANOVA) y a los efectos significativos se les realizó una prueba de promedios de Tukey con nivel significancia del 95\% (P $\leq$ 0,05), utilizando el Software Statistica versión 7.0 (Statsoft Inc. 2005). 


\section{RESULTADOS Y DISCUSIÓN}

\section{Porcentaje de Postura}

El análisis estadístico indicó que hubo diferencias significativas entre los tratamientos $(P \leq 0,05)$. La variación en el porcentaje de huevos se debe a la adición de harina de pescado, evidenciando que aumenta más de $10 \%$ en todos los casos que se adicionó el complemento nutricional rico en proteína, esto al compararlos con el tratamiento control (tabla 2). Según Pinto et al. (2002) existe una correlación positiva entre la concentración de proteína en la dieta suministrada y el consumo del alimento con la postura de los huevos de codornices. El consumo final de los diferentes tratamientos fue aproximadamente de $24 \mathrm{~g} /$ día.

\begin{tabular}{|c|c|c|c|c|c|c|c|c|}
\hline \multirow[t]{2}{*}{ PARÁMETROS } & \multicolumn{8}{|c|}{ TRATAMIENTOS } \\
\hline & T1 & T6 & T7 & & T8 & & T9 & T10 \\
\hline $\begin{array}{l}\text { Postura } \\
\text { día) }\end{array}$ & $\begin{array}{l}81,00 \mathrm{a} \\
1,2\end{array}$ & $\begin{array}{l}92,00 \mathrm{ab} \\
3,4\end{array}$ & $\begin{array}{l}91,67 \mathrm{ab} \\
3,1\end{array}$ & \pm & $\begin{array}{l}96,33 b \\
2,5\end{array}$ & \pm & $\begin{array}{l}103,33 b \pm \\
2,9\end{array}$ & $100 \mathrm{~b} \pm 3,0$ \\
\hline Huevos rotos (\%) & $\begin{array}{l}31,86 \mathrm{a} \quad \pm \\
1,7\end{array}$ & $\begin{array}{l}25,46 b \\
0,8\end{array}$ & $\begin{array}{l}17,44 \mathrm{c} \\
1,0\end{array}$ & & $\begin{array}{l}14,45 \mathrm{c} \\
1,3\end{array}$ & \pm & $\begin{array}{l}10,13 \mathrm{~d} \quad \pm \\
1,5\end{array}$ & $\begin{array}{l}26,18 c \\
0,9\end{array}$ \\
\hline $\begin{array}{l}\text { Producción comer- } \\
\text { cializable (\%) }\end{array}$ & $\begin{array}{l}68,14 \mathrm{a} \\
1,5\end{array}$ & $\begin{array}{l}74,54 \mathrm{a} \\
1,0\end{array}$ & $\begin{array}{l}82,56 b \\
0,7\end{array}$ & \pm & $\begin{array}{l}85,55 b \\
1,3\end{array}$ & \pm & $\begin{array}{l}89,87 \mathrm{C} \\
0,9\end{array}$ & $\begin{array}{l}73,82 \mathrm{~b} \\
1,0\end{array}$ \\
\hline Peso (g) & $9,01 \mathrm{a} \pm 1,0$ & $8,28 \mathrm{a} \pm 0$, & $\begin{array}{l}9,06 a \\
0,4\end{array}$ & & $\begin{array}{l}9,78 \mathrm{a} \\
0,3\end{array}$ & \pm & $8,54 a \pm 0,9$ & $\begin{array}{l}8,44 \mathrm{a} \\
0,6\end{array}$ \\
\hline Volumen (cm3) & $8,20 \mathrm{a} \pm 1,0$ & $6,04 \mathrm{a} \pm 1,3$ & $\begin{array}{l}8,09 a \\
0,9\end{array}$ & \pm & $\begin{array}{l}6,32 \mathrm{a} \\
1,7\end{array}$ & \pm & $7,53 a \pm 1,5$ & $\begin{array}{l}6,57 a \\
1,2\end{array}$ \\
\hline
\end{tabular}

Tabla 2. Comportamiento de producción, peso e volumen de los huevos de codorniz en las diferentes dosis de harina de pescado.

Media \pm desviación estándar. Valores con letras distintas en la misma fila presentan diferencia ( $\mathrm{P} \leq 0,05)$. T1: alimento comercial al 100\%; T6: alimento comercial $+6 \%$ de harina de pescado; $\mathrm{T} 7$ : alimento comercial $+7 \%$ de harina de pescado; T8: alimento comercial $+8 \%$ de harina de pescado; T9: alimento comercial $+9 \%$ de harina de pescado; T10: alimento comercial + $10 \%$ de harina de pescado.

\section{Porcentaje de Huevos Rotos}

Se observaron diferencias significativas entre los tratamientos $(P \leq 0,05)$ (tabla 2), donde la muestra T1 presentó el mayor porcentaje de huevos rotos. Lo que se relaciona posiblemente por no presentar un adecuado suministro de proteína y también se puede atribuir este factor al picoteo de las aves. En cuanto al tratamiento T10, este resultó con el mayor porcentaje de huevos rotos, posiblemente se debe a un elevado porcentaje de harina pescado, que influencia en el tamaño del huevo y grosor de la cascara. Estos resultados son similares a los reportados por Rosario y Nieves (2015), que determinaron mayor porcentaje de huevos rotos en el caso de inclusión de $12 \%$ de harina de residuos aserrados de carnicerías en la dieta de las codornices. Sin embargo, la reducción en el grosor de la cáscara está asociada al hecho que las aves 
producen huevos grandes y con cáscara más fina con el pasar del tiempo; además de posibles deficiencias nutricionales (Moras, 2008; Moura et al., 2009; Rosario y Nieves, 2015).

\section{Producción de Huevos}

El análisis estadístico mostró diferencias significativas entre tratamientos ( $\mathrm{P}$ $\leq 0,05$ ) (tabla 2), observándose una mayor producción de huevos en codornices alimentadas con harina de pescado. El tratamiento con $9 \%$ de harina de pescado presentó la mayor producción de huevos de buena calidad (tabla 2). La producción de huevos fue similar a la registrada por Pinto et al. (2002); Freitas et al. (2005) obtenida en codornices en la fase inicial de postura. De acuerdo con estos resultados se evidencia que la harina de pescado hasta 9 $\%$, es una excelente opción para preparar alimentos balanceados para codornices en postura con el propósito de aumentar la producción de huevos.

\section{Peso del Huevo}

El análisis de varianza mostró que no hubo diferencia significativa en la variable peso del huevo de codorniz, encontrando valores promedio entre 8,28 y $9.78 \mathrm{~g}$ (tabla 2). El rango de valores de peso observados en este estudio, son similares a los encontrados con otros tratamientos de alimentación para la producción de huevos con fines comerciales (Costa et al., 2011; Hurtado-Nery et al., 2015).

\section{El Volumen del Huevo}

No hubo diferencias significativas entre los tratamientos en cuanto al promedio del volumen del huevo de codorniz. Los resultados oscilan entre 6,04 a $8,20 \mathrm{~cm} 3$, siendo el tratamiento testigo el que presentó el mayor valor promedio (tabla 2). Se evidencia por los resultados que el suplemento de harina de pescado no influye en el volumen del huevo. Así bien, es importante resaltar que el volumen de los huevos se mantuvo semejante en todos los tratamientos, alcanzando el mayor valor el tratamiento T7 (tabla 2). Los resultados encontrados en esta investigación, coinciden con lo señalado por Quintana (1993), quien indica que el volumen del huevo es afectado por factores como, alimentación del ave y temperatura del lugar donde se encuentra tanto el ave como el huevo. En este sentido, se determinó que los alimentos utilizados en este ensayo no presentan problemas de ausencia de aminoácidos y presencia de compuestos antinutricionales, ya que no hubo influencias negativas en la variable volumen del huevo.

\section{Manchas/Pigmentación de los Huevos}

Los resultados indican que hubo diferencia sobre huevos de codorniz poco manchados (pigmentación grisácea clara), por lo que fue evidente la influencia en el consumo de alimento en esta etapa. Para los huevos medianamente manchados no hubo diferencia entre los tratamientos (tabla 3), encontrando valores idénticos para los tratamientos T7 y T10. En el caso de los huevos manchados hubo diferencia, encontrando que el tratamiento T9 presentó el mayor valor (41\%), reflejando un efecto positivo de la harina de pescado 
como complemento nutricional en la calidad de la pigmentación del huevo de codorniz. De esta forma, los huevos del tratamiento T9 presentaron color oscuro, una mezcla de pigmentos marrones y grises con una base negra oscura. Esta cualidad, define estos huevos como excelentes para la reproducción. De acuerdo con las observaciones de Galíndez et al. (2009), los huevos con mayor número de manchas permanecen por más tiempo en el tracto reproductivo de la hembra, por lo tanto, se presenta una cascara de mayor grosor y cantidad de cutina sobre los poros, lo que aumenta la posibilidad de un mejor desarrollo embrionario.

\begin{tabular}{|c|c|c|c|c|c|c|}
\hline \multirow{2}{*}{$\begin{array}{l}\text { CARACTERIZACIÓN } \\
\text { CUALITATIVA }\end{array}$} & \multicolumn{6}{|c|}{ TRATAMIENTOS } \\
\hline & $\mathrm{T} 1$ & T6 & T7 & T8 & T9 & $\mathrm{T} 10$ \\
\hline \multicolumn{7}{|l|}{ Mancha } \\
\hline Poco manchados & $17 \mathrm{f}$ & $22 d$ & $18 \mathrm{e}$ & $24 b$ & $25 a$ & $24 c$ \\
\hline $\begin{array}{l}\text { Medianamente } \\
\text { manchados }\end{array}$ & $28 \mathrm{a}$ & $28 \mathrm{a}$ & $32 \mathrm{a}$ & $28 a$ & $29 a$ & $32 \mathrm{a}$ \\
\hline Muy manchados & $31 \mathrm{f}$ & $36 \mathrm{~d}$ & $39 c$ & $35 \mathrm{e}$ & $41 \mathrm{a}$ & $40 \mathrm{~b}$ \\
\hline \multicolumn{7}{|l|}{ Brillo } \\
\hline Opacos & $41 \mathrm{e}$ & $47 \mathrm{c}$ & $50 \mathrm{~b}$ & $55 \mathrm{a}$ & $37 f$ & $42 d$ \\
\hline Brillantez & $34 f$ & $35 e$ & $41 \mathrm{c}$ & $39 d$ & $61 \mathrm{a}$ & $57 b$ \\
\hline
\end{tabular}

Tabla 3. Valores promedios de las variables mancha y brillo de los huevos de codorniz.

Media. Valores con letras distintas en la misma fila presentan diferencia. T1: alimento comercial al 100\%; T6: alimento comercial $+6 \%$ de harina de pescado; T7: alimento comercial + 7\% de harina de pescado; T8: alimento comercial $+8 \%$ de harina de pescado; T9: alimento comercial $+9 \%$ de harina de pescado; T10: alimento comercial $+10 \%$ de harina de pescado.

Los tratamientos con adición de harina de pescado, registraron mayor presencia de manchas en los huevos cuando éstos se comparan con T1, lo que evidencia la importancia de la alimentación rica en proteína en el color, es decir, pigmentación del huevo. La pigmentación corresponde a una membrana que forma la cutícula de la cáscara, reflejándose en el huevo de codorniz por manchas de color marrón oscuro distribuidas homogéneamente por toda la superficie. Según Galíndez et al. (2009) los huevos cuya cáscara tiene mayor cantidad de manchas tienen mayor importancia para la reproducción, pues estos reflejan ventaja en eclosión.

\section{Brillo de los Huevos}

Hubo diferencias entre los tratamientos en cuanto al promedio de las características opacas y brillantez de los huevos de codornices. Se evidencia que el suministro nutricional tiene efecto en el brillo de los huevos. Se observó que el valor promedio en huevos opacos más alto corresponde al tratamiento T8 con $55 \%$; sin embargo, para el promedio de la variable brillantez el tratamiento T9 presentó el mayor valor promedio (tabla 3). Por otro lado, se pudo comprobar que las muestras del T9, presentaron características determinantes a la hora de seleccionar el huevo fértil para la reproducción artificial, como lo son: manchado, brillantez y uniformidad. Galíndez et al. (2009), 
encontró que los huevos más brillantes mantienen un menor intercambio gaseoso y de humedad con el medio ambiente que los huevos opacos, debido a la presencia de una película protectora que los recubre. En consecuencia, estos huevos conservan mejor la integridad del albumen y tienen mayor porcentaje de eclosión. En este particular, Hurtado-Nery et al. (2013) recomienda que en la alimentación de codornices de postura se puede utilizar hasta un $20 \%$ de material sólido de escama de pescado como fuente alternativa de calcio sin afectar los parámetros de calidad interna y externa del huevo.

\section{Análisis Bromatológico de los Huevos}

Los huevos de codorniz de los diferentes tratamientos con harina de pescado presentaron diferencias significativas $(\mathrm{P} \leq 0.05)$ comparándolo con el tratamiento control. Se debe destacar, que los huevos a partir de codornices alimentadas con las dietas de harina de pescado presentaron (tabla 4) alto contenido de proteínas seguido del contenido de lípidos, fibra y cenizas, en comparación con T1. Con estos resultados estimados en composición g/100 g de porción comestible, se ha demostrado la importancia que tiene el componente nutricional en la alimentación de las codornices, que permite aumentar simultáneamente la producción y contenido proteico de los huevos.

\begin{tabular}{|c|c|c|c|c|c|c|}
\hline \multirow{2}{*}{$\begin{array}{l}\text { A N Á L I S I S } \\
(\%)\end{array}$} & \multicolumn{6}{|c|}{ TRATAMIENTOS } \\
\hline & T1 & T6 & T7 & T8 & T9 & T10 \\
\hline Proteínas & $\begin{array}{l}17,50 a \pm \\
0,05\end{array}$ & $\begin{array}{l}18,00 b \pm \\
0,04\end{array}$ & $\begin{array}{l}18,70 \mathrm{~d} \\
\pm 0,05\end{array}$ & $\begin{array}{l}19,00 \mathrm{c} \\
\pm 0,06\end{array}$ & $\begin{array}{l}21,50 \mathrm{~b} \pm \\
0,04\end{array}$ & $\begin{array}{l}24,00 \mathrm{a} \pm \\
0,05\end{array}$ \\
\hline Lípido & $\begin{array}{l}3,80 \mathrm{a} \pm \\
0,23\end{array}$ & $\begin{array}{l}7,14 \mathrm{c} \pm \\
0,34\end{array}$ & $\begin{array}{l}5,90 \mathrm{~d} \pm \\
0,41\end{array}$ & $\begin{array}{l}5,30 \mathrm{e} \pm \\
0,36\end{array}$ & $\begin{array}{l}4,90 \mathrm{e} \pm \\
0,23\end{array}$ & $\begin{array}{l}9,80 b \pm \\
0,50\end{array}$ \\
\hline Fibra & $\begin{array}{l}3,70 \mathrm{a} \pm \\
0,18\end{array}$ & $\begin{array}{l}2,40 \mathrm{c} \\
\pm 0,14\end{array}$ & $\begin{array}{l}1,26 \mathrm{~d} \\
\pm 0,20\end{array}$ & $\begin{array}{l}1,14 \mathrm{e} \\
\pm 0,17\end{array}$ & $\begin{array}{l}1,21 \mathrm{~d} \\
\pm 0,19\end{array}$ & $\begin{array}{l}2,82 \mathrm{~b} \\
\pm 0,21\end{array}$ \\
\hline \multirow[t]{3}{*}{ Ceniza } & $\begin{array}{l}9,54 \mathrm{a} \pm \\
0,07\end{array}$ & $\begin{array}{l}12,56 b \pm \\
0,07\end{array}$ & $\begin{array}{l}12,16 \mathrm{c} \\
\pm 0,04\end{array}$ & $\begin{array}{l}10,28 \mathrm{~d} \\
\pm 0,06\end{array}$ & $\begin{array}{l}12,56 \mathrm{e} \\
\pm 0,04\end{array}$ & $\begin{array}{l}13,36 \mathrm{f} \\
\pm 0,05\end{array}$ \\
\hline & \multicolumn{6}{|c|}{ Huevos } \\
\hline & \multicolumn{3}{|c|}{ Control } & \multicolumn{3}{|c|}{ Harina de pescado } \\
\hline Proteínas & \multicolumn{3}{|c|}{$10,20 \mathrm{a} \pm 0,24$} & \multicolumn{3}{|c|}{$13,05 b \pm 0,20$} \\
\hline Lípido & \multicolumn{3}{|c|}{$9,03 a \pm 0,30$} & \multicolumn{3}{|c|}{$12,00 \mathrm{~b} \pm 0,35$} \\
\hline Fibra & \multicolumn{3}{|c|}{$1,29 a \pm 0,21$} & \multicolumn{3}{|c|}{$2,29 b \pm 0,24$} \\
\hline Ceniza & \multicolumn{3}{|c|}{$2,00 \mathrm{a} \pm 1,40$} & \multicolumn{3}{|c|}{$3,30 \mathrm{~b} \pm 0,16$} \\
\hline
\end{tabular}

Tabla 4. Análisis bromatológico tanto de los tratamientos con diferentes dosis de harina de pescado como de los huevos

Media \pm desviación estándar. Valores con letras distintas en la misma fila presentan diferencia (P $\leq$ 0,05). T1: alimento comercial al 100\%; T6: alimento comercial $+6 \%$ de harina de pescado; $\mathrm{T} 7$ : alimento comercial $+7 \%$ de harina de pescado; T8: alimento comercial + 8\% de harina de pescado; T9: alimento comercial $+9 \%$ de harina de pescado; T10: alimento comercial + $10 \%$ de harina de pescado.

El contenido de proteína y lípidos totales del huevo de codorniz fue semejante a lo obtenido por González y Hernández (2011) para huevo de codorniz, con valores de 13,63 y 12,59 \% respectivamente. Así mismo, Lima et al. (2014); Hurtado-Nery et al. (2015); Shim y Vohra (1984), recomiendan que los 
valores de proteína bruta en los huevos de codorniz japónica deben oscilar entre 24 y $27 \%$ durante las primeras tres semanas de vida, y entre 17 y $22 \%$ desde la tercera semana hasta el final del ciclo de producción. El valor óptimo para la dieta de la codorniz fue observado en el T9 con 21,50 \% de proteína en la harina de pescado (tabla 4). En otros estudios, se ha demostrado que la calidad de huevos de codornices no se ve afectada, si se proporcionan dietas con niveles de 17,5 y 22 \% de proteína bruta (Hurtado-Nery et al., 2015).

\section{Análisis sensorial}

Los huevos de codorniz de los diferentes tratamientos no presentaron diferencias significativas $(P \leq 0,05)$ entre ellos, es decir, la adición de harina de pescado en la dieta de las codornices no influyo en los parámetros sensoriales evaluados. Se debe destacar, que los huevos no adquirieron ni sabor ni olor a pescado, atributos importantes para la aceptación por parte de los consumidores. Los resultados de esta investigación son similares a los obtenidos por Carranco et al. (2011) y Cornejo et al. (2008) quienes adicionaron harina y aceite de pescado al concentrado alimenticio de gallinas ponedoras y no encontraron rechazos organolépticos en los huevos.

\section{Conclusiones}

El tratamiento T9, permitió obtener la mayor cantidad de huevos, con menor proporción de huevos rotos, valores razonables de peso y volumen, y una adecuada composición proximal.

Se evidenció que existe influencia positiva de la adición de harina de pescado en la calidad del manchado y brillantez del huevo de codorniz, resultando T9 como el más apropiado.

En relación a los atributos sensoriales de los huevos, no fue reportado por los consumidores que el producto presentara flavor a pescado.

\section{Agradecimientos}

Los autores agradecen a la Universidad Politécnica Territorial del Oeste de Sucre "Clodosbaldo Russian", Cariaco, estado Sucre, Venezuela, por el apoyo ofrecido durante el desarrollo del experimento. 


\section{REFERENCIAS BIBLIOGRÁFICAS}

AOAC. (2000). Official methods of analysis of the association of official analytical chemists, HORWITZ, W, 17a ed. Arlington: AOAC Inc.

Carranco, M.E., Calvo, C.C., Carrillo, D.S., Ramírez, C.R., Morales, B.E., Sanginés, G.L., Fuente, M.B., Ávila, G.E., \& Pérez-Gil, R.F. (2011). Harina de crustáceos en raciones de gallinas ponedoras. Efecto en las variables productivas y evaluación sensorial de huevos almacenados en diferentes condiciones. Revista Cubana de Ciencia Agrícola, 45(2):171-175.

Cornejo, S., Hidalgo, H., Araya, J., \& Pokniak, J. (2008). Suplementación de dietas de gallinas de postura comercial con aceites de pescado de diferentes grados de refinación. Efectos productivos en las aves y en la calidad organoléptica de los huevos. Archivos de Medicina Veterinaria, 40(1): 4550. Disponible en http://dx.doi.org/10.4067/S0301-732X2008000100006

Costa, F.G.P., Brandão, P.A., Souza, J.G., da-Silva, J.H.V., Goulart, C. de C., \& Rabello, C.B.V. (2011). Exigência de cálcio para codornas japonesas (Coturnix coturnix japonica) machos na fase de 1 a 21 dias de idade. Ciência e Agrotecnologia, 35(2): 410-414..

Dutcosky S.D. (2013). Análises sensorial de alimentos. (4th ed.). Curitiba: Champagnat.

Freitas, A.C de., de-Fátima, F.F.M., Freitas, E.R., Sucupira, F.S., \& de-Oliveira, B.C.M. (2005). Efeito de níveis de proteína bruta e de energia metabolizável na dieta sobre o desempenho de codornas de postura. Revista Brasileira de Zootecnia, 34(3): 838-846.

Galíndez, R., de Basilio, V, de Martínez, G., Vargas, D., Uztariz, E., \& Mejía, P. (2009). Evaluación de la fertilidad y eclosión en la codorniz japonesa. Zootecnia Tropical, 27(1): 7-15.

González, F.J.S., \& Hernández, U.A. (2011). Evaluación sensorial de huevos de codorniz en conserva y composición nutrimental. Revista Electronica de Veterinaria, 12(8): 1-10.

Hurtado-Nery, V.L., Corredor, L.F., \& Torres, D.M. (2010). Grano de soya integral cocido en la alimentación de codornices. Orinoquia, 14(1): 27-32. http://orinoquia.unillanos.edu.co/index.php/orinoquia/article/ view/123/579

Hurtado-Nery, V.L., Herrera, Y.M., \& Gómez, D.A. (2013). Efecto del uso de la escama de pescado en la alimentación de codornices sobre la calidad del huevo. Revista Citecsa, 4(6): 59-69. Disponible en http://www.unipaz.edu. co/ojs/index.php/revcitecsa/article/view/49

Hurtado-Nery, V.L., Torres-novoa, D.M., \& Daza-Garzón, M.F. (2015). Efectos de la proteína bruta y energía metabolizable sobre la calidad del huevo de codorniz. Orinoquia, 9(2): 195. Disponible en http://www.scielo.org. co/scielo.php?pid=S0121-37092015000200006\&script=sci_abstract\&tln$\mathrm{g}=\mathrm{es}$ 
INE - Instituto Nacional de Estadística. (2011). Informe geoambiental estado sucre, República Bolivariana de Venezuela, p. 185. Disponible en http:// www.ine.gov.ve/index.php?option=com_content\&view=category\&i$\mathrm{d}=68 \&$ Itemid $=49 \#$

Lima, R.C., Costa, F.G.P., Goulart, C.C., Cavalcante, L.E., Freitas, E.R., Silva, J.H.V., Dantas, L.S., \& Rodrigues, V.P. (2014). Exigência nutricional de proteína bruta para codornas japonesas (Coturnix coturnix japonica) na fase de postura. Arq Bras Med Vet Zootec, 66(4): 1234-1242. Disponible en http://www.scielo.br/scielo.php?script=sci_arttext\&pi$\mathrm{d}=$ S0102-09352014000401234

Lucotte, G. (1985). La Codorniz: cría y explotación. Mundi - Prensa. Madrid, España.

Moras, M.C.R de las. (2008). Alteraciones de la cáscara, clara y yema de huevo. Ganadería, (52): 56-57. Completar la cita

Moura, A.M.A de., da-Trindade, R.N.S.R., Fonseca, J.B., Mendonça, V.R.A., \& Hurtado, N.V.L. (2009). Efecto de diferentes niveles dietéticos de lisina total sobre la calidad del huevo de codornices japonesas (Coturnix japonica). Archivos Latinoamericanos de Producción Animal, 17(3-4): 67-75. Disponible en http://www.bioline.org.br/pdf?la09010

Oliveira, I.S de., Lourenço, L.de F.H., Sousa, C.L., Peixoto, M.R.S.J, \& Ribeiro, S.D.C.D.A. (2015). Composition of MSM from Brazilian catfish and technological properties of fish flour. Food Control, 50: 38-44. Disponible en https://www.sciencedirect.com/science/article/pii/S0956713514004678

Pereira, A.A., Ferreira, D.A., Júnior, D.N.G., Lima, C.B., de Moura, A.S., \& de Lima, D.M.J. (2016). Raspa da mandioca para codornas em postura. Acta Veterinaria Brasilica, 10(2): 123-129.

Pérez \& Pérez. (1974). Coturnicultura, Trabajo de Cría y Explotación Industrial de Codornices. 2º ed. Científica - Médica. Barcelona, España. 500p.

Pino Hernández, E.J.G., Carvalho, R.N.D., Joele, M.R.S.P., Araújo, C.D.S., \& Lourenço, L.D.F.H. (2017). Effects of modified atmosphere packing over the shelf life of sous vide from captive pirarucu (Arapaima gigas). Innovative Food Science \& Emerging Technologies, 39: 94-100. Disponible en https://www.sciencedirect.com/science/article/pii/S1466856416306889

Pinto, R., Ferreira, A.S., Albino, L.F.T., Gomes, P.C., \& Júnior, J.G.de V. (2002). Níveis de proteína e energia para codornas japonesas em postura. Revista Brasileira de Zootecnia, 31(4): 1761-1770. Completar datos

Quintana, J. (1993). Avitécnia: Manejo de las aves domésticas más comunes. Trillas ( $3^{\circ}$ ed). México, D.F.

Quitral, V., Donoso, M.L., \& Acevedo, N. (2009). Comparación físico-química y sensorial de huevos de campo, orgánicos y comerciales. Revista de Salud Pública y Nutrición, 10(2): 4.

Ribeiro, C.L.N., Barreto, S.L.T., Reis, R.S., Muniz, J.C.L., Viana, G.S., Mendonça, M.O., Silva, L.M., \& Mencalha, R. (2015). Utilização de farinha de casca de ovos em dietas para codornizes japonesas na fase de 11 a 25 semanas de idade. Revista de Ciências Agrárias, 38(1): 11-17. 
Rosario, J., \& Nieves, D. (2015). Producción y calidad de huevos de codornices alimentadas con dietas con harina de residuos aserrados de carnicerías. Revista Científica, FCV-LUZ, XXV (2): 139-144. Disponible en http:// www.redalyc.org/articulo.oa?id=95935857008

Shim, K.F., \& Vohra, P. (1984). A review of the nutrition of japanese quail. Worlds Poult SciJ, 40(3): 261-74. Disponible en https://www.cambridge.org/ core/journals/world-s-poultry-science-journal/article/review-of-the-nutrition-of-japanese-quail/30F9EE91AD56CAD38AF3105C36F7C262

\section{Cumbres}

